\title{
Masticatory Functional Load increases ACTN2 and ACTN3 mRNA and $\alpha$-Actinin-2 Protein Expression in Rat Masseter Muscle
}

\author{
Nur Masita Silviana $^{1}$, Sri Andarini $^{2}$, Diana Lyrawati $^{3}$, Mohammad Hidayat $^{4}$ \\ ${ }^{1}$ Doctoral Program in Biomedical Science, Faculty of Medicine, Brawijaya University, \\ Malang, Indonesia \\ ${ }^{2}$ Department of Public Health, Faculty of Medicine, Brawijaya University, Malang, Indonesia \\ ${ }^{3}$ Department of Pharmacy, Faculty of Medicine, Brawijaya University, Malang, Indonesia \\ ${ }^{4}$ Department of Orthopedics, Syaiful Anwar General Hospital, Faculty of Medicine, \\ Brawijaya University, Malang, Indonesia
}

INTRODUCTION: $\alpha$-Actinins play a structural and the regulatory roles in the cytoskeletal organization. It forms a lattice structure that secure together the actin containing thin filaments which generate and transmit muscle contractile forces. The morphological and biochemical of rat masseter muscles are known to change the reactions to masticatory functional load, but the effect to the $\alpha$-actinins is not known. This study was to determine the response of $\alpha$-actinins to the masticatory functional load

METHODS: Twenty-four male Wistar rats aged 3 weeks were randomly divided into three groups of liquid (LD group), soft (SD group) and hard (HD group) diets, then in the end of eight-week the rats were sacrificed. In order to study the effect of masticatory functional load on mRNA Actn 2 and Actn 3 and the protein expression of $\alpha$-actinin- 2 and $\alpha$-actinin-3, central portion of the superficial masseter muscles was examined.

RESULTS: The mRNA expression of $A \operatorname{ctn} 2$ and $A c t n 3$ and protein expression of $\alpha$-actinin-2 of HD group significantly higher than LD group as control.

DISCUSSION AND CONCLUSION: These results suggest that masticatory functional load organizes the expression of $A \operatorname{ctn} 2$ and $A c t n 3$ mRNA and $\alpha$-actinin-2 protein in the rat masseter muscle by relevant stimuli during muscle physiological adaptation.

Keywords: $\alpha$,, -actinins, masseter muscles, masticatory function

Nur Masita Silviana,

nmsilviana@gmail.com

0000-0003-2981-1061

$+62-341-551611$

\section{Introduction}

There is a direct causal relation between transformations of masticatory muscle function, induced by changing the diet consistency, and muscle changes in a complete masticatory system in animal study. Kiliaridis and Shyu reported that masticatory muscle strength after tetanic stimulation was lower with soft diet food rather than hard diet food. ${ }^{1}$ Satellite cell population is smaller in masseter muscle with reduced masticatory function. ${ }^{2}$ Decreasing of physical consistency of the diet can increase type IIB fibers, fiber diameter and muscle mass and cross-sectional area. ${ }^{3-7}$ The animals masseter muscles that had been fed with soft diet food have smaller proportion and cross-sectional area of fibers co-expressing myosin heavy chain (MyHC)-I and MyHC-cardiac alpha than those in controls. ${ }^{8}$ 
The $\alpha$-Actinins which belongs to actinin binding proteins group, is classified into muscle and non-muscle isoforms. $\alpha$-Actinins cross-linked actin filaments are located on the sarcomere $\mathrm{Z}$ disk, to help stabilization and architecture of muscle contraction in skeletal muscle..$^{9} \alpha$ Actinins participate in wide-ranging signal transduction complexes by interacting with other proteins to accelerate physiological changes. ${ }^{10}$ It considered as important structural component associated with the contractile muscle of force generation and transmission as in regular myofibrillar arrays maintenance. $\alpha$-Actinins have two isoforms of the skeletal muscle namely $\alpha$-actinin- 2 and $\alpha$-actinin-3. ${ }^{11-13} \alpha$-Actinin- 2 is indicated within all muscle fibers, including cardiac muscle and brain, while $\alpha$-actinin-3 is only restricted to most type II fast contracting fibers. Both of actinins initiate energy production of force-generating glycolytic at high speed.

Through interaction with calcineurin signaling, $\alpha$-actinin-3, which encoded by Actn 3 gene, can contribute in muscle function to influence the fiber types proportion during the growth ${ }^{14}$ $\alpha$-Actinin-3 deficiency (XX) may affect the decrease of muscle strength, power and enhanced endurance performance in elite athletes and general population. ${ }^{15}$ Ogura et al. claimed that the total amount of $\alpha$-actinin-2 protein level increased in plantaris and in white and red gastrocnemius muscles, but no significant disparity seen in $\alpha$-actinin- 3 expression following exercise training. ${ }^{16}$ Khaledi et al. have found that actinins $n$ RNA and protein levels transform as the response to progressive resistance training. ${ }^{17}$ This showed that after 8 weeks of exercise in female Sprague-Dawley rats, both $\alpha$-actinin- 2 and -3 mRNA levels were upregulated. The protein expression of $\alpha$-actinin- 2 had significant enhancement in the training group although no difference seen in $\alpha$-actinin-3 protein expression. ${ }^{17}$

There are not many reports that link $\alpha$-actinins to masseter muscle activity. Zebrick et al. stated that the level of masseter muscle mRNA expression was different for single nucleotide polymorphism genotypes of Actn3. ${ }^{18}$ Significantly, the frequency of Actn3 genotypes was different in sagittal and vertical classifications of malocclusion. In skeletal class II malocclusion, the clearest association was the enhancement of 577XX genotype. This genotype also produced smaller diameters of fast type II fibers in masseter muscles. ${ }^{18}$ These suggest that Actn3 genotype may affect some aspects in muscle function, such as $\alpha$-actinin-3 in enhancing the forceful and fast skeletal muscle contraction. The deficiency of $\alpha$-actinin-3 showed the need of $\alpha$-actinin-3 for optimal forceful and rapid muscle contractions.

However, the response of $\alpha$-actinins in masticatory muscle is still unknown. The contraction velocity and the maximum force generation of jaw-closing muscle that responsible while chewing, are influenced by the decrease of the masticatory functional load during its development. Our study aims to examine the effect of masticatory functional load on the expression of Actn2 and Actn3 mRNA and proteins in masseter muscle rat.

\section{Materials and Methods}

A total of twenty-four 3-week-old male Wistar rats (approximately 60g in body weight) were randomly classified into three groups, which contained for 8 rats in each group. Group 1, as a control group, was placed into a liquid diet (LD group) and fed with a blended mixture of pellets and water with ratio of 1:4. Group 2 was placed into a soft diet (SD group) and fed with shurry mixture of pellets softened in water with 1:1 ratio. Group 3 was fed with regular rat pellets and composed in hard diet (HD group). All groups were fed ad libitum and given water. The rats were put in cage separately in suspended metal cages with no other material or object that could lead masticatory stimulus inside the cages. Every week, the body weight and physical condition were measured and recorded due to monitor the rats condition. After 8 weeks of examination, all rats were sacrificed using exsanguination under anesthesia with pentobarbital sodium at a fetal overdose of $50 \mathrm{mg} / \mathrm{kg}$. Then, the central portion of the superficial masseter muscles in rats was immediately dissected and frozen in a liquid nitrogen 
and stored at $-85^{\circ} \mathrm{C}$. This experimental protocol was approved by the Ethics Committee for Animal Research of Universitas Brawijaya, Malang, Indonesia (269/EC/KEPK/07/2017).

\section{Actn2 and Actn3 mRNA expression analysis}

RNA isolation was performed using Total RNA Purification Kit (Jena Bioscience, Jena, Thuringia, Germany).cDNA was synthesized using iScript ${ }^{\mathrm{TM}}$ cDNA Synthesis Kit (Bio-Rad, Hercules, California, USA). The condition for synthetizing cDNA was: priming for 5 min at $25^{\circ} \mathrm{C}$, reverse transcription for 20 minutes at $46^{\circ} \mathrm{C}$, enzyme inactivation for 1 minute at $95^{\circ} \mathrm{C}$. Quantitative reverse transcription real time PCR (qRT-PCR) was conducted using SsoFast ${ }^{T}$ EvaGreen ${ }^{\circledR}$ Supermix (Bio-Rad, Hercules, California, USA). The sequences of the primer were as follow: Actn2 F: 5'-CTATTGGGGCTGAAGAAATCGTC-3' and Actn2 R: 5', CTGAGATGTCCTGAATGGCG-3'. Actn3 F: 5'-AGAAACAGCAGCGGAAAACC-3' and Actn3 R: 5'-CAGGGCTTTGTTGACATTG-3' ${ }^{17} \beta$-Actin was selected for the normalization of quantitative data, with the primers sequences as follow: $\beta$-Actin $\mathrm{F}$ : 5 '-

ACCATGTACCCAGGCATTGC-3' and $\beta$-Actin R: 5'-CACACAGAGTACTTGCGCTC$3,{ }^{17}$ Actn 3 amplification was performed in condition: enzyme activation for 3 minutes at $95^{\circ} \mathrm{C}$, denaturation at $95^{\circ} \mathrm{C}$ for $5 \mathrm{sec}$, annealing at $54.9^{\circ} \mathrm{C}$ for 1 minute $(45 \mathrm{cycles}$ ); Actn2 and $\beta$-actin: enzyme activation for 3 min at $95^{\circ} \mathrm{C}$, denaturation at $95^{\circ} \mathrm{C}$ for $5 \mathrm{sec}$, annealing at $57,3^{\circ} \mathrm{C}$ for 1 minute (45 cycles). For all qPCR experiments comparative quantitation measured by CFX96 ${ }^{\mathrm{TM}}$ Real-Time PCR Detection System (Bio-Rad, Hercules, California,USA).

\section{$\alpha$-Actinin-2 and $\alpha$-Actinin-3 protein expression analysis}

Protein expression analysis was performed using western blot method with $\alpha$-actinin-2 [N1N3] and $\alpha$-actinin-3 antibody (GeneTex, Irvine, California, USA). Protein bands in the gel were transferred to PVDF membrane for overnight, followed by blocking in skim milk for $1 \mathrm{~h}$. membrane was incubated in 1:1000 anti-actinin antibody diluted in pbs-skim milk $1 \%$ for overnight. Secondary antibody was added the day after and BCIP/NBT was used as substrate. Bands were analyzed using Quantity One Analysis Software (Bio-Rad, Hercules, California, USA).

\section{Statistical analysis}

The data are presented with the mean + SEM (the standard error of the mean) with statistical analysis using One-way ANOVA with the Tukey's post hoc test. The statically significant standard is $\mathrm{P}<0.05$.

\section{Results}

The result showed that there was no significant difference found in rat body weight at week 8 among three experimental groups (see Table 1). The mean body weight of the rat in LD group was $179.88 \pm 0.48$, in SD group was $179.30 \pm 0.75$, and 179.64 \pm 0.72 in HD group.

\section{mRNA Expression of Actn2 and Actn3}

The levels of the Actn2 and Actn3 mRNA expressions in the three experimental groups were assessed using qPCR. In HD group, the Actn2 and Actn3 mRNA levels from the masseter muscle were significantly increased by mean factors 2.29 (SEM=0.41) and 2.19 (SEM=0.2) respectively, in contrast with the LD group as the control group. In SD group, even though the expression of Actn2 and Actn3 mRNA up-regulated, but there was no significant difference was detected compared to LD and HD group $(\mathrm{P}<0.005)$ (Figure 1).

\section{Protein Expression of $\alpha$-actinin-2 and $\alpha$-actinin-3}


The effect of masticatory muscle function on $\alpha$-actinin- 2 and $\alpha$-actinin- 3 proteins expression in the three experimental groups were assessed using western blot. It was found greater level of $\alpha$-actinin- 2 in the HD group (18.45, SEM=0.78) as compared to LD group(14.40, SEM=0.44), significantly (Figure 2A). Meanwhile, protein levels of $\alpha$-actinin-3 in the HD group also increased compared to the SD and LD groups, by mean factors 16.75 (SEM=0.72), $14.16(\mathrm{SEM}=0.91)$ and $14.66(\mathrm{SEM}=0.97)$ respectively. However, there was no significant difference of $\alpha$-actinin-3 between the three groups (Figure 2B).

\section{Discussion}

This study investigated functional influence of masticatory muscles with consistency of diet variation of Actn 2 and Actn 3 mRNA and $\alpha$-actinin-2 and $\alpha$-actinin-3 proteins expression The results showed that in three experimental groups, no transformation showed in masticatory pattern in response to the consistency of diet variations. Also, there were no significant body weight differences between LD, SD and HD groups during 8 weeks of the entire experimental period. It found that both Actn2 and Actn3 mRNA are up regulated along with increase consistency of diet in the SD and HD group as compared to LD group as control group. The increasing of $\alpha$-actinin-2 expression happened along with increase consistency of diet. However, there was significant differences in the levels of Actn2, Actn3 and $\alpha$-actinin- 2 expression in LD group compared to HD group. The difference is not significant when the LD group and HD group are compare to the SD group. Meanwhile, in $\alpha$-actinin-3, there were no significant differences among three groups.

The previous study showed that in about $16-18 \%$ of the global population indicated that the lack of $\alpha$-actinin-3 due to homozygosis for the common null of Actn3 577X polymorphism may occurs. ${ }^{19}$ This is also associated in untrained adolescents, elite athletes and young adults with low sprint performance and muscle strength and power. ${ }^{13}$ In human skeletal muscle the total protein level of $\alpha$-actinins decreases after irregular exercise and recovers systematically in 7-8 days after exercise is complete. ${ }^{20}$ This may suggested that $\alpha$-actinin- 3 considered as important structural component for optimal forceful and rapid muscle contractions. ${ }^{13}$

The results of this study are similar to several animal studies performed to study the effect of physiological stimuli on cellular $\alpha$-actinins. Khaledi et al. examined the progressive resistance training effects on Actn 3 and Actn3 genes and proteins level. They found that mRNA levels of Actn 2 and Actn 3 and $\alpha$-actinin-2 increased but no transformation was found in $\alpha$-actinin-3 protein expression. ${ }^{17}$ Ogura et al. observed the effects of endurance exercise training on both $\alpha$-actinin- 2 and $\alpha$-actinin-3 levels in a rat model. After exercise for 8 weeks on a treadmill, $\alpha$-actinin- 2 expression in the plantaris muscles was slightly higher than $\alpha$ actinin- $3 .^{21}$ They also demonstrated that $\alpha$-actinin isoforms could respond to other physiological stimuli. Theresult showed that $\alpha$-actinin-3 expression was slightly higher than $\alpha$-actinin-2 after hind limb unloading. ${ }^{22}$

The diet food consistency in laboratory changes the demands of biting strength level, masticatory activity and behavior, and these in turn convert the composition and diameter of the fiber types in the animal masticatory muscles. ${ }^{1,3-5}$ In this study, physiological stimulation through masticatory functional load shows that cellular $\alpha$-actinins is involved in masseter muscle. Zebrick et al. used the masseter muscle of orthognathic surgery to examined Actn2 and Actn 3 expression and genetic variation, then determined their associations with musculoskeletal malocclusion phenotypes. Masseter muscle samples from 60 orthognathic surgery-subjects included the following vertical and sagittal classifications such as; Class II and Class III open bite, Class II and Class III deep bite, and Class II and Class III normal bite malocclusions. Their results demonstrated that Actn3 polymorphism, R577X, was related to Class II and deep-bite skeletal malocclusions. The loss of $\alpha$-actinin-3 was accompanied by smaller diameter of type II fiber in masseter muscles. The experiments of real-time 
polymerase chain reaction indicated that Actn3 mRNA expression was almost cannot be detected with 577XX genotype and Actn2 expression levels remained unchanged. It suggested that in masseter muscles, Actn2 may not compensate for the loss of $\alpha$-actinin- $3 .^{18}$ The adoption of a consistency of diet variations is related to the muscle fiber type alterations of histological, morphological and biochemical. In the softer diet group, there was small percentage of type IIA fibers and large percentage of type IIB fibers in anterior deep masseter compared to the normal diet group. ${ }^{1}$ Fundamentally, $\alpha$-actinin- 2 is expressed in all skeletal muscle fibers, while $\alpha$-actinin-3 expression is restricted to type II fast-contracting skeletal muscle fibers. ${ }^{11,13}$ Ogura et al. indicated along $\alpha$-actinin-3 expression increased, relative content of type II MyHC and fast myosin levels also enhanced after the hind limb unloading. Exercise training has function to change MyHC from IIb to IIa. Their study suggested that changes in MyHC composition may be affected to the enhancement of aerobic capacity for skeletal muscle after training. ${ }^{22}$ The line of fiber type specific gene expression activated $\alpha$ Actinin-3 which define the type and size of fibers by binding to calsarcin family signaling protein on $\mathrm{Z}$ disk, that in turn, it binds to signaling protein calcineurin. ${ }^{23,24}$

This masticatory functional load showed that there were similar reactions as in skeletal muscle models. This showed that during masticatory muscle function, there was expression alteration on Actn 2 and Actn 3 mRNA and $\alpha$-actinin- 2 protein expressions. Actn 2 and Actn3 mRNA and $\alpha$-actinin- 2 significantly alters the expression along with increased masticatory functional load between HD and LD groups. Non-significant differences were shown in the SD group in contrast to the LD and HD groups. This is likely to indicate that the masticatory of functional load in the SD group insufficient to induce mRNA and protein expression. However, further investigation on the differences in Actn 2 and Actn 3 numbers between LD, SD and HD Group which increased in time or remained stable is needed.

In conclusion, Actn 2 and Actn 3 mRNA and $\alpha$-actinin- 2 protein are set in rat masseter muscle along with the increasing of masticatory functional load. Even though this seems that cellular $\alpha$-actinins of the masseter muscle adapt the function changes, the exact mechanism still needs to be clarified.

\section{Acknowledgement}

The authors gratefully thank you for the financial assistance received from the Directorate General of Strengthening for Research and Development, the Ministry of Research, Technology and Higher Education through a Doctoral Research grant No: 338.178 / UN10.C10 / PN / 2018.

\section{References}

1. Kiliaridis $\mathrm{S}$, Shy $\mathrm{BC}$. Isometric muscle tension generated by masseter stimulation after prolonged alteration of the consistency of the diet fed to growing rats. Arch Oral Biol. 1988;33(7):467-472. doi:10.1016/0003-9969(88)90026-x

2. Kuijpers MAR, Grefte S, Bronkhorst EM, Carels CEL, Kiliaridis S, Von den Hoff JW. Reduced masticatory function is related to lower satellite cell numbers in masseter muscle. Eur J Orthod. 2014;36(3):262-267. doi:10.1093/ejo/cjs044

3. Kiliaridis S, Engström C, Thilander B. Histochemical analysis of masticatory muscle in the growing rat after prolonged alteration in the consistency of the diet. Arch Oral Biol. 1988;33(3):187-193. doi:10.1016/0003-9969(88)90044-1

4. He T, Olsson S, Daugaard JR, Kiliaridis S. Functional influence of masticatory muscles on the fibre characteristics and capillary distribution in growing ferrets (Mustela putonusfuro) - A histochemical analysis. Arch Oral Biol. 2004;49(12):983-989. doi:10.1016/j.archoralbio.2004.07.011

5. Kitagawa Y, Mitera K, Ogasawara T, Nojyo Y, Miyauchi K, Sano K. Alterations in 
enzyme histochemical characteristics of the masseter muscle caused by long-term soft diet in growing rabbits. Oral Dis. 2004;10(5):271-276. doi:10.1111/j.1601-0825.2004.01020.x

6. Saito T, Fukui K, Akutsu S, et al. Effects of diet consistency on the expression of insulin-like growth factors (IGFs), IGF receptors and IGF binding proteins during the development of rat masseter muscle soon after weaning. Arch Oral Biol. 2004; 49(10):777782. doi:10.1016/j.archoralbio.2004.02.014

7. Urushiyama T, Akutsu S, Miyazaki J-I, Fukui T, Diekwisch TGH, Yamane A. Change from a hard to soft diet alters the expression of insulin-like growth factors, their receptors, and binding proteins in association with atrophy in adult mouse masseter muscle Cell Tissue Res. 2004;315(1):97-105. doi:10.1007/s00441-003-0787-0

8. Vreeke M, Langenbach GEJ, Korfage JAM, Zentner A, Grünheid T. The masticatory system under varying functional load. Part 1: Structural adaptation of rabbit jaw muscles to reduced masticatory load. Eur J Orthod. 2011;33(4):359-364. doi:10.1093/ejo/cjq083

9. Mills M, Yang N, Weinberger R, et al. Differential expression of the actin-binding proteins, alpha-actinin-2 and -3, in different species: implications for the evolution of functional redundancy. Hum Mol Genet. 2001;10(13):1335-1346.

doi:10.1093/hmg/10.13.1335

10. Djinovic-Carugo K, Gautel M, Ylänne J, Young P. The spectrin repeat: a structural platform for cytoskeletal protein assemblies. FEBS Lett. 2002;513(1):119-123.

doi:10.1016/s0014-5793(01)03304-X

11. MacArthur DG, North KN. A gene for speed? The evolution and function of alphaactinin-3. Bioessays. 2004;26(7):786-795. doi:10.1002/bies.20061

12. MacArthur DG, North KN. ACTN3: A genetic influence on muscle function and athletic performance. Exerc Sport Sci Rev. 2007;35(1):30-34.

doi:10.1097/JES.0b013e31802d8874

13. North KN, Beggs AH. Deficiency of a skeletal muscle isoform of alpha-actinin (alpha-actinin-3) in merosin-positive congenital muscular dystrophy. Neuromuscul Disord. 1996;6(4):229-235. doi:10.1016/0960-8966(96)00361-6

14. Vincent B, De Bock K, Ramaekers M, et al. ACTN3 (R577X) genotype is associated with fiber type distribution. Physiol Genomics. 2007;32(1):58-63.

doi:10.1152/physiolgenomics.00173.2007

15. Yang N, Garton F, North K. alpha-actinin-3 and performance. Med Sport Sci. 2009;54:88-101. doi:10.1159/000235698

16. Ogura Y, Naito H, Kakigi R, et al. Different adaptations of alpha-actinin isoforms to exercise training in rat skeletal muscles. Acta Physiol. 2009;196(3):341-349.

doi:10.1111/j.1748-1716.2008.01945.x

17. khaledi N, Fayazmilani R, Gaeini AA, Javeri A. Progressive Resistance Training Modulates the Expression of ACTN2 and ACTN3 Genes and Proteins in the Skeletal Muscles. Am J Sport Sci Med. 2016;4(2):26-32. doi:10.12691/ajssm-4-2-1

18. Zebrick B, Teeramongkolgul T, Nicot R, et al. ACTN3 R577X genotypes associate

with Class II and deepbite malocclusions. Am J Orthod Dentofac Orthop. 2014;146(5):603611. doi:10.1016/j.ajodo.2014.07.021

19. North KN, Yang N, Wattanasirichaigoon D, Mills M, Easteal S, Beggs AH. A common nonsense mutation results in $\alpha$-actinin-3 deficiency in the general population. Nat Genet. 1999;21(4):353-354. doi:10.1038/7675

20. Yu J-G, Fürst DO, Thornell L-E. The mode of myofibril remodelling in human skeletal muscle affected by DOMS induced by eccentric contractions. Histochem Cell Biol. 2003;119(5):383-393. doi:10.1007/s00418-003-0522-7

21. Ogura Y, Naito H, Kakigi R, et al. Effects of ageing and endurance exercise training on alpha-actinin isoforms in rat plantaris muscle. Acta Physiol. 2011;202(4):683-690. 
doi:10.1111/j.1748-1716.2011.02284.x

22. Ogura Y, Naito H, Kakigi R, Ichinoseki-Sekine N, Kurosaka M, Katamoto S. Alphaactinin-3 levels increase concomitantly with fast fibers in rat soleus muscle. Biochem Biophys Res Commun. 2008;372(4):584-588. doi:10.1016/j.bbrc.2008.05.059

23. Frey N, Olson EN. Calsarcin-3, a novel skeletal muscle-specific member of the calsarcin family, interacts with multiple Z-disc proteins. J Biol Chem. 2002;277(16):1399814004. doi:10.1074/jbc.M200712200

24. Swoap SJ, Hunter RB, Stevenson EJ, et al. The calcineurin-NFAT pathway and muscle fiber-type gene expression. Am J Physiol Cell Physiol. 2000;279(4):C915-924. doi:10.1152/ajpcell.2000.279.4.C915 


\section{Table and Figure}

Table 1. The data are presented mean body weight (grams) \pm standard deviation (SD) in liquid, soft and hard diet groups, for 8 weeks of experiment ( $\mathrm{n}=8$ per group).

\begin{tabular}{lllll} 
Week & $2^{\text {nd }}$ & $4^{\text {th }}$ & $6^{\text {th }}$ & $8^{\text {th }}$ \\
\hline Diet & Mean + SD & Mean + SD & Mean \pm SD & Mean + SD \\
\hline Liquid & $79.06 \pm 0.26$ & $136.91 \pm 0.18$ & $158.31 \pm 0.49$ & $179.88 \pm 0.48$ \\
\hline Soft & $78.64 \pm 0.47$ & $136.5 \pm 0.46$ & $157.89 \pm 0.45$ & $179.30 \pm 0.75$ \\
\hline Hard & $78.96 \pm 0.19$ & $136.95 \pm 0.21$ & $158.51 \pm 0.52$ & $179.64 \pm 0.72$ \\
\hline
\end{tabular}

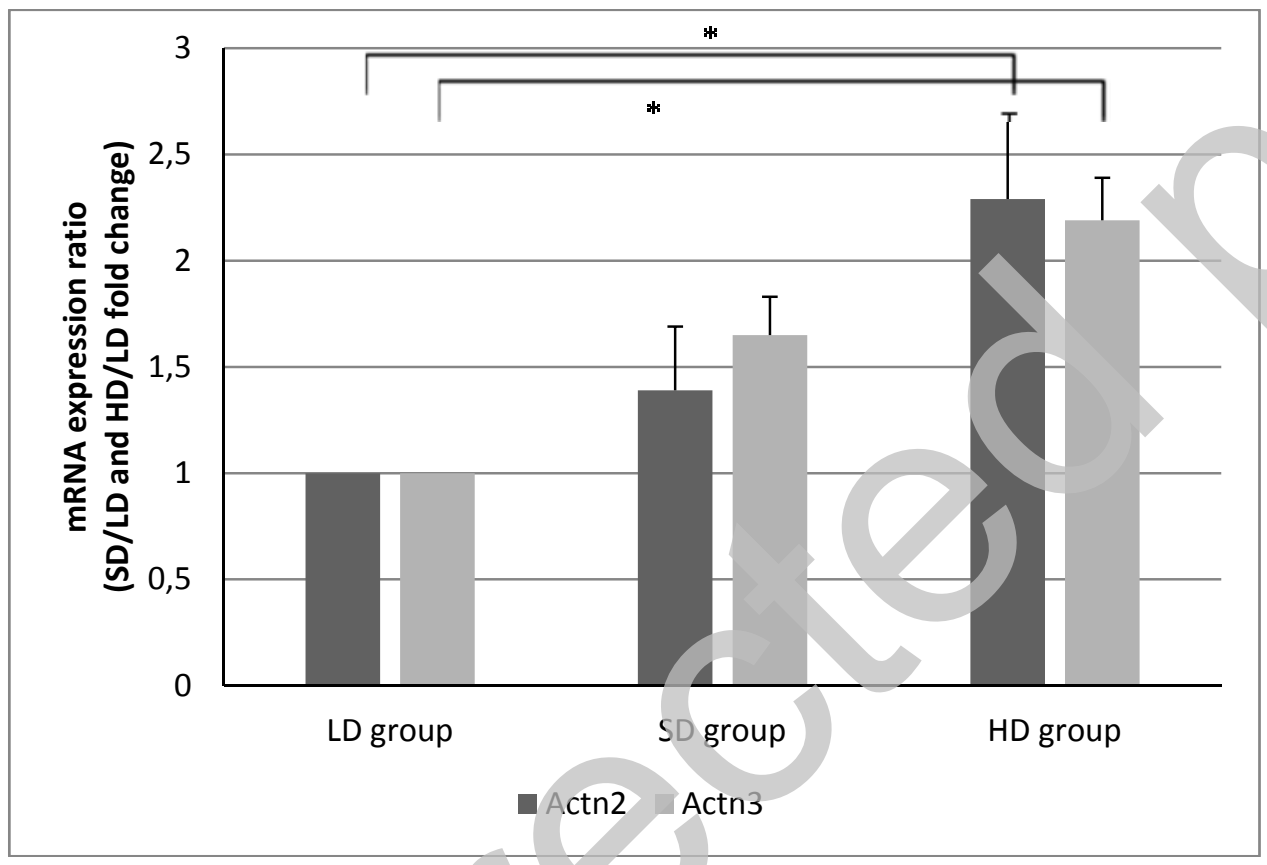

Figure 1. Expression of Actn2 and Actn3 mRNA in masseter muscle shown as fold change compared to the liquid diet/LD group as the control group. Statistical analysis is tested using One-way ANOVA with the Tukey's post hoc test. * Significant with $\mathrm{P}<0.05$.

\section{A}

\section{$\alpha-$ Actinin}

\section{$\beta$-Actin}




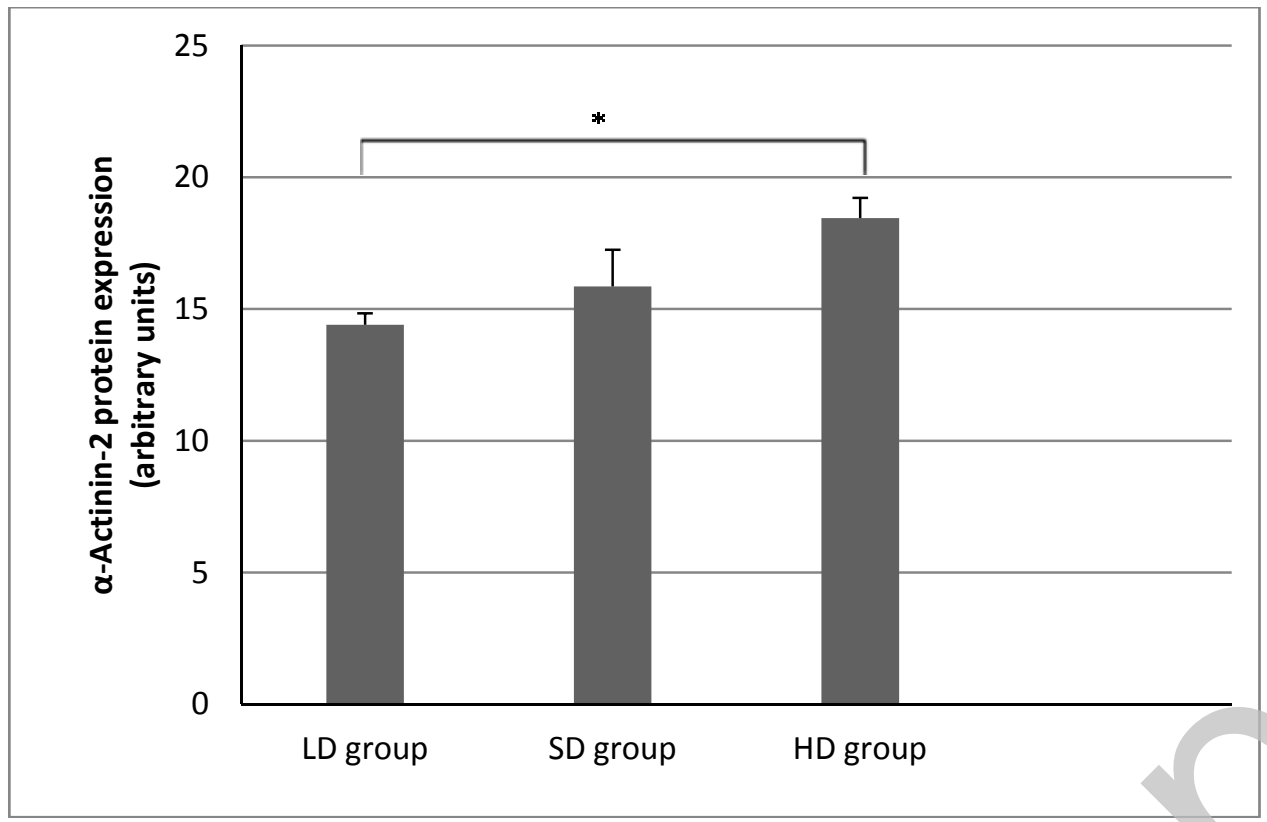

B

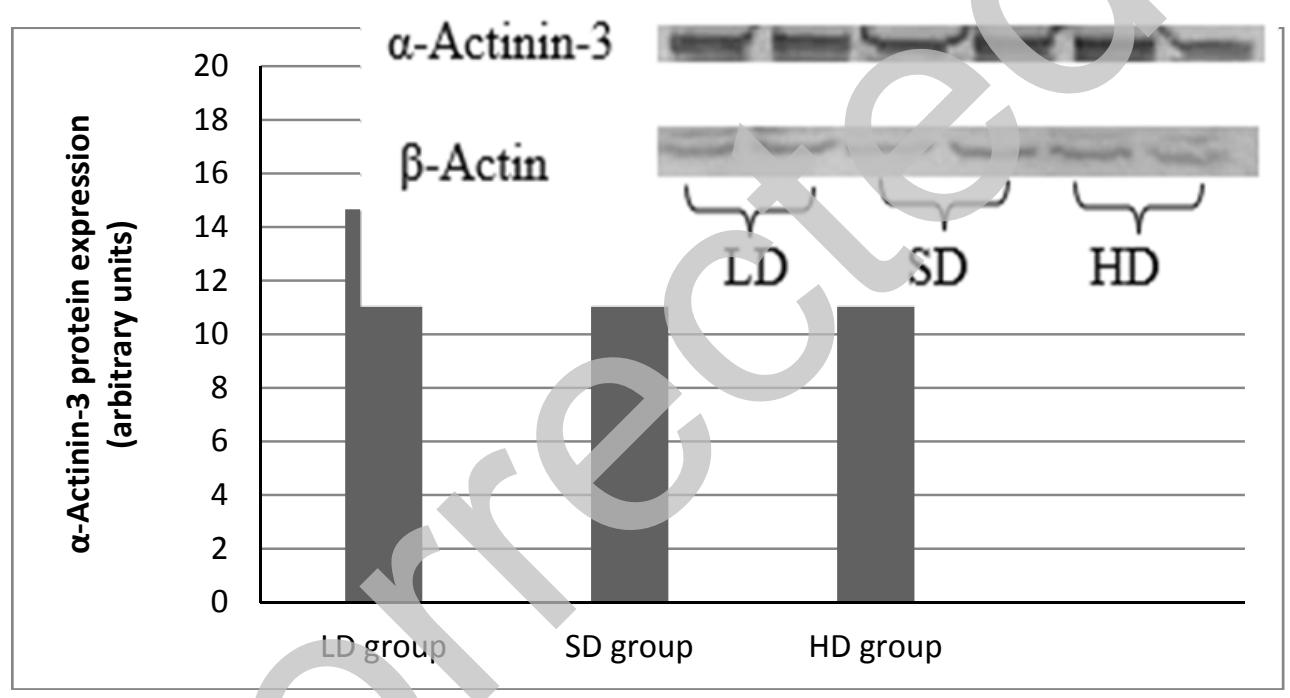

Figure 2. Western blot analysis.A. $\alpha$-Actinin-2 and B. $\alpha$-actinin-3 proteins expression in the LD, SD and HD groups. $\beta$-actin was used as sample loading control. The bar graphs on the right represent means \pm SEM. *Significant with $p<0.05$. 


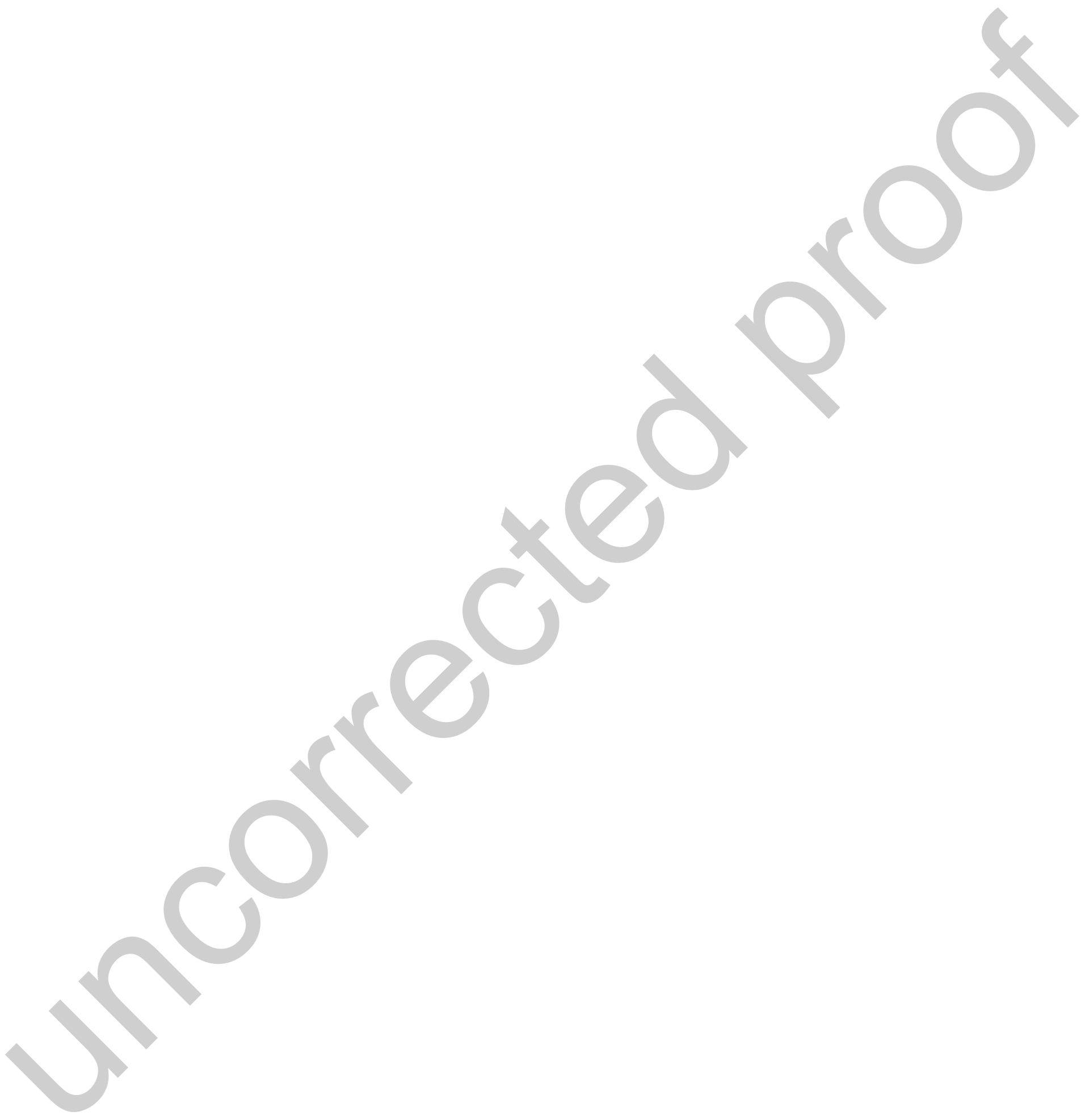

